Rev. Mus. Argentino Cienc. Nat., n.s.

8(1): 63-68, 2006

Buenos Aires, ISSN 1514-5158

\title{
Humerus of a basal abelisauroid theropod from the Late Cretaceous of Patagonia
}

\author{
Fernando E. NOVAS ${ }^{1,2}$, Martín D. EZCURRA ${ }^{1}$ \& Federico L. AGNOLIN ${ }^{1}$ \\ ${ }^{1}$ Laboratorio de Anatomía Comparada y Evolución de los Vertebrados, Museo Argentino de Ciencias Naturales \\ «Bernardino Rivadavia», Av. Angel Gallardo 470, Buenos Aires (1405), Argentina. ${ }^{2}$ CONICET, \\ email: fernovas@yahoo.com.ar.
}

\begin{abstract}
Here we describe a partial humerus of a medium-sized theropod dinosaur from the Upper Cretaceous (Late Turonian - Early Coniacian) Portezuelo Formation, Neuquén Province, Argentina. The humerus shares with Abelisauroidea and Elaphrosaurus an articular head proximally oriented, and deltopectoral crest strongly reduced. The bone is referred to Abelisauroidea on the basis of the following derived features: proximal tuberosity on caudal surface of humeral shaft, and greater tubercle at level with the medial tuberosity. The gracile morphology of the humerus, as well as the poor development of its medial tuberosity, resemble more Masiakasaurus than the condition present in abelisaurids (e.g., Carnotaurus, Aucasaurus), in which the humerus is more robust and the medial tuber-osity is prominent. Available information demonstrates that during deposition of the Portezuelo Formation large and bulky abelisaurids (e.g., Ekrixinatosaurus) lived together with gracile and medium-sized abelisauroids.
\end{abstract}

Key words: Abelisauroidea, Upper Cretaceous, Patagonia, Argentina.

Abelisauroids constitute a group of predatory dinosaurs that were highly diversified and widely dispersed in Gondwana during the Cretaceous (e.g., Bonaparte, 1986; Sereno et al., 2004). Abelisauroidea is usually splited into two clades (Bonaparte, 1991): the small to medium-sized noasaurids, including

Noasaurus, Masiakasaurus, Laevisuchus and Deltadromeus (Carrano et al., 2002; Sereno et al., 2004), and the medium to large-sized abelisaurids represented, among others by Rugops, Carnotaurus, Abelisaurus, Ekrixinatosaurus, and

Aucasaurus (Bonaparte, 1985; Bonaparte \& Novas, 1985; Bonaparte et al., 1990; Coria et al., 2002; Calvo et al., 2004a; Sereno et al., 2004).

The theropod record of the Upper Cretaceous Portezuelo Formation (Late Turonian-Early Coniacian; Leanza et al., 2004) currently consists of the abelisaurid Ekrixinatosaurus novasi (Calvo et al., 2004a), the basal tetanuran Megaraptor namunhuaiquii (Novas, 1998; Calvo et al., 2004b), the maniraptoran alvarezsaurid Patagonykus puertai (Novas, 1997), two yet undescribed coelurosaurians (Coria \& Currie, 2002; Porfiri et al., 2005), and the dromaeosaurid maniraptorans Unenlagia comahuensis (Novas \& Puerta, 1997), U. paynemili (Calvo et al., 2005), and Neuquenraptor argentinus (Novas \& Pol, 2005). Here we describe a partial humerus that constitutes the first non-abelisaurid abelisauroid recorded in the Portezuelo Formation. This new find improves our knowledge of the humeral morphology of basal abelisauroids.

\section{MATERIAL AND METHODS}

Institutional abbreviations. MACN-CH, Museo Argentino de Ciencias Naturales "B. Rivadavia", Colección Chubut. MCF-PVPH, Museo Carmen Funes, Paleontología de Vertebrados, Plaza Huincul, Neuquén, Argentina.

Systematic nomenclature. We follow Sereno et al. (2004) and Rauhut (2003), regard-ing the phylogenetic relationships of Abeli-sauroidea within Ceratosauria. Abelisauroidea was defined by Holtz (1994) as a stem-based clade that includes all theropods closer to Carnotaurus sastrei than to Ceratosaurus nasicornis, thus Elaphrosaurus bambergi Janensch, 1920 and the specimen described here belong to this clade.

\section{SYSTEMATIC PALEONTOLOGY}

Theropoda Marsh, 1881

Neotheropoda Bakker, 1986

Ceratosauria Marsh, 1884

Abelisauroidea Bonaparte, 1991

Genus et sp. indet.

Material. MCF-PVPH 53, proximal half of a right humerus. 


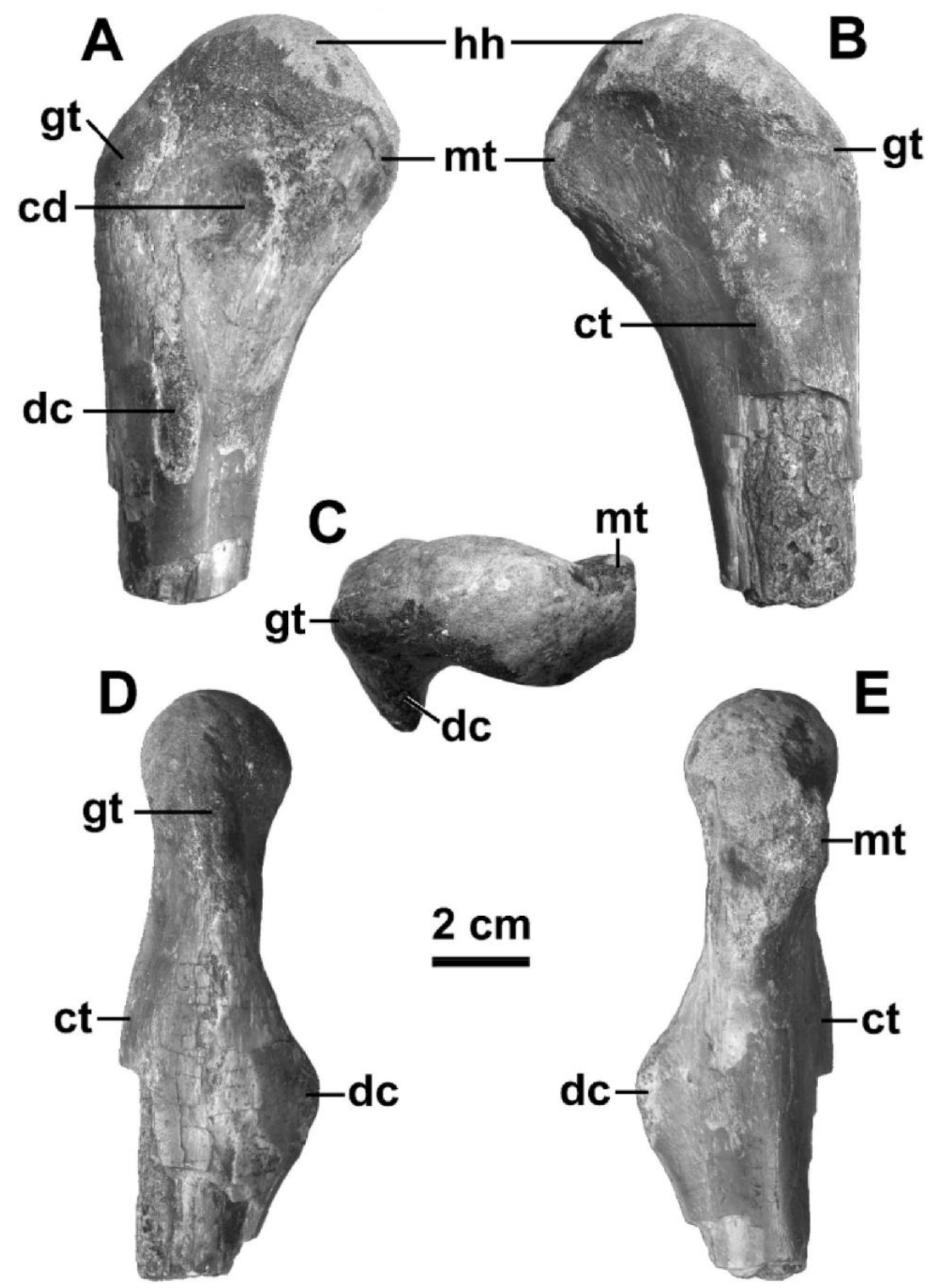

Fig. 1. MCF-PVPH 53 humerus. A) cranial, B) caudal, C) proximal, D) lateral, and E) medial views. Abbreviations: cd. coracobrachial depression, ct. caudal tuberosity dc. deltopectoral crest, gt. greater tubercle, hh. humeral head, mt. medial tuberosity. Scale bar $2 \mathrm{~cm}$. 

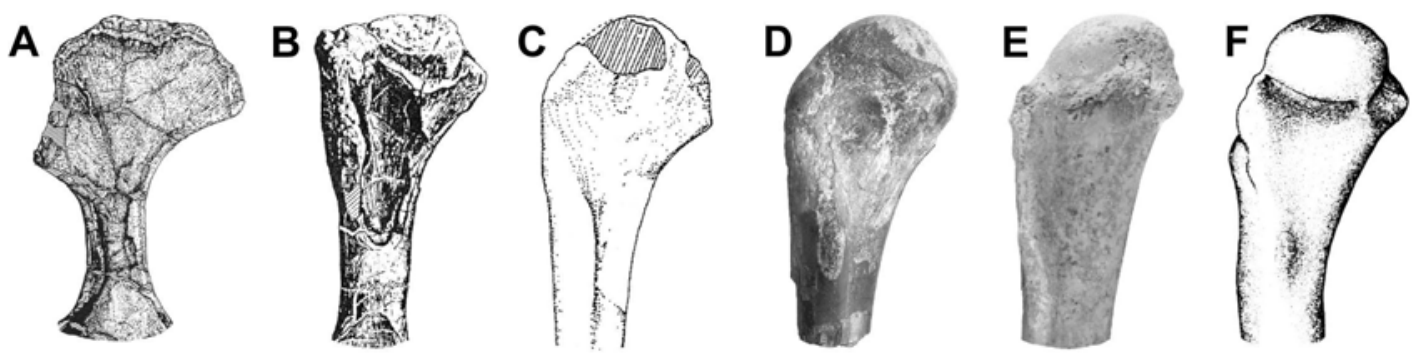

Fig. 2. Humeral proximal halves of several theropods in cranial view. A) Baryonyx (from Charig \& Milner, 1986), B) Ceratosaurus (from Madsen \& Wells, 2000), C) Elaphrosaurus (from Galton, 1982), D) MCF-PVPH 53, E) Masiakasaurus (from Carrano et al., 2002) and F) Carnotaurus (from Bonaparte et al., 1990). Not to scale.
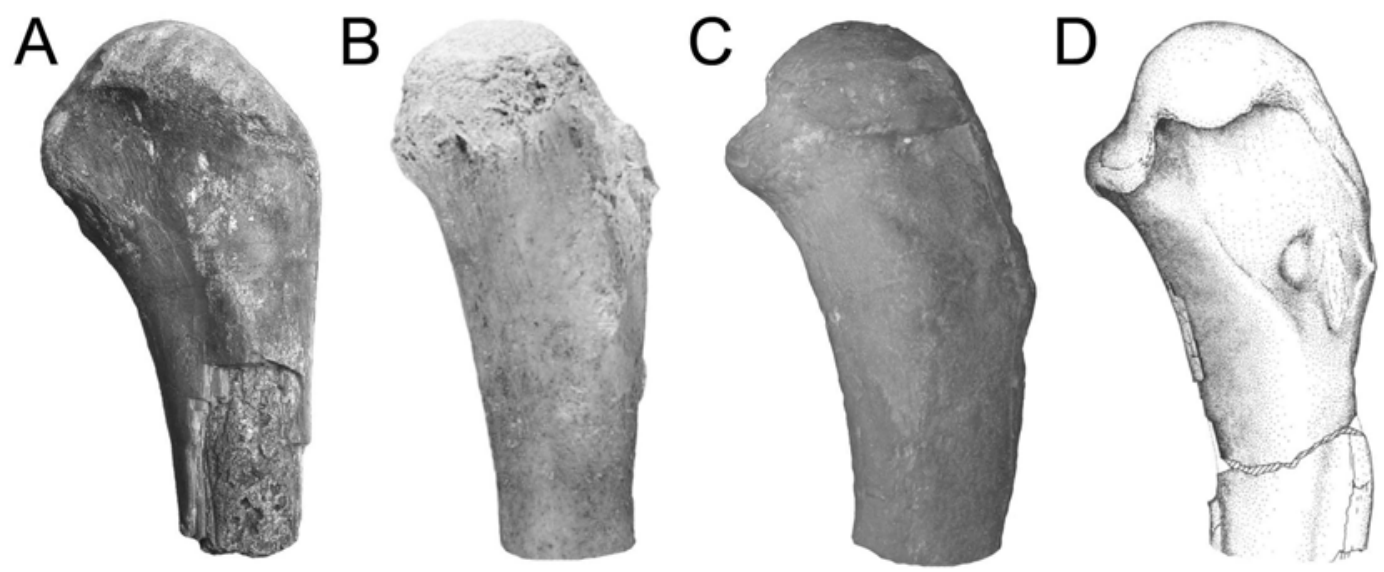

Fig. 3. Humeral proximal halves of several abelisauroids in caudal view. A) MCF-PVPH 53, B) Masiakasaurus (from Carrano et al., 2002), C) Carnotaurus (MACN-CH 894) and D) Aucasaurus (from Coria et al., 2002). Not to scale.

Locality and horizon. The material was collected at Sierra del Portezuelo, $20 \mathrm{~km}$ West from Plaza Huincul, Neuquén Province, Argentina, from levels corresponding to the Portezuelo Formation.

\section{Description}

The preserved portion of humerus (Fig. 1) closely matches the morphology of the Malagasy abelisauroid Masiakasaurus knopfleri (Carrano et al., 2002), thus suggesting that the bone, as in this dinosaur, was long and slender when complete. On the basis of the maximum proximal width of humerus $(5.1 \mathrm{~cm})$, we estimate that the whole length of the bone was roughly $30 \mathrm{~cm}$.

The humeral head is rounded and proximally inflated, resembling the basal ceratosaurian Elaphrosaurus (Galton, 1982) and the abelisauroids Carnotaurus, Aucasaurus, and Masiakasaurus (Figs. 2-4). This condition distin- guishes MCF-PVPH 53 (together with the four taxa cited before) from the remaining theropods (e.g., Syntarsus, Ceratosaurus, Allosaurus, Deinonychus), in which the humeral head is kidned-shaped (in proximal view) and flattened (in cranial aspect). However, MCF-PVPH 53 has a humeral head that looks more plesiomorphic than Carnotaurus and Masiakasaurus, in being craniocaudally more compressed than in the later two taxa (Fig. 4).

In MCF-PVPH 53 the greater tubercle is located on the proximolateral corner of humerus; it is reduced and located at level with the inter-nal tuberosity, as it occurs in other abelisauroids (e.g., Masiakasaurus, Aucasaurus, Carnotaurus). This condition differs from that of basal theropods (e.g., Syntarsus), basal ceratosaurians (e.g., Ceratosaurus) and most tetanurans (e.g., Allosaurus, Deinonychus), in which the greater tubercle is more prominent and more proximally 

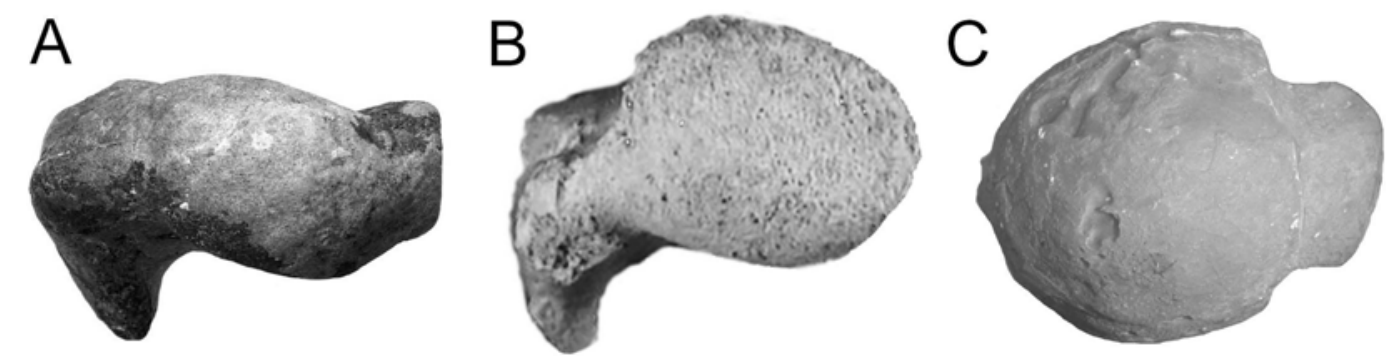

Fig. 4. Humeri of several abelisauroids in proximal view. A) MCF- PVPH 53, B) Masiakasaurus (from Carrano et al., 2002) and C) Carnotaurus (MACN-CH 894). Not to scale.

located with respect to the internal tuberosity. In Elaphrosaurus the greater tubercle is weakly developed (as in abelisauroids), but it is located more proximally as in Theropoda ancestrally.

In MCF-PVPH 53 the internal tuberosity is highly reduced, as it occurs in Masiakasaurus and probably also in Elaphrosaurus. However, this condition is not uniformly present among abelisauroids because in abelisaurids (e.g.,

Aucasaurus, Carnotaurus) the internal tuberos-ity is prominent and conical-shaped (Fig. 2). A similar condition to that of Abelisauridae is present in most theropods, including Herrerasaurus (Sereno, 1993), coelophysoids (e.g.,

Syntarsus; Raath, 1977), Dilophosaurus (Welles, 1984), and basal tetanurans ( e.g., Allosaurus, Acroncanthosaurus; Madsen, 1976; Currie \& Carpenter, 2000).

On the cranial surface of humeral shaft, and immediately distal to the humeral head, a shallow sulcus is present, presumably for attachment of the acrocoracohumeral ligament (Baumel \& Raikow, 1993). The shallow condition of this sulcus contrasts with the deep ligament groove present in Carnotaurus and Masiakasaurus. Congruently, the caudal surface of MCF-PVPH 53 is devoid of a sulcus below the humeral head, resembling Masiakasaurus but contrasting with

Carnotaurus and Aucasaurus, in which a deep groove is present.

In MCF-PVPH 53 the deltopectoral crest is strongly reduced and triangular in side view, being transversely thick and cranially projected. This peculiar condition is shared with other abelisauroids (e.g., Masiakasaurus, Carnotaurus), albeit at least some of these features (i.e., deltopectoral crest reduced and triangular shaped) are also present in Elaphrosaurus. MCFPVPH 53 differs from Carnotaurus in that in the latter the deltopectoral crest is even more reduced.

On the caudal surface of the humeral shaft, MCF-PVPH 53 bears a longitudinal and conspicu- ous tuberosity. On the caudolateral edge of the bone, a smaller and lesser developed second tuberosity is present. Thus, the caudal surface of the humerus exhibits a double prominence located almost at level with the proximal end of the deltopectoral crest. This caudal tuberosity is documented also in Aucasaurus and Carnotaurus. In Masiakasaurus a prominence is visible, but its double condition can not be discerned (Carrano et al., 2002; fig. 11). The condition in Ceratosaurus is inconclusive (Madsen \&Welles, 2000), but in coelophysoids (e.g., Syntarsus, Liliensternus) and basal tetanurans (e.g.,

Baryonyx, Torvosaurus, Allosaurus, Deinonychus) this caudal tuberosity is lacking.

\section{CONCLUSIONS}

The humerus of abelisaurid theropods is well represented by Carnotaurus and Aucasaurus. The same bone, however, remains poorly known in nonabelisaurid abelisauroids, the main source of information being currently restricted to the noasaurid Masiakasaurus (Carrano et al., 2002; due to the highly fragmentary condition of the holotype specimen of Deltadromeus agilis Sereno et al., 1996, and the few available descriptions and illustrations of the forelimb bones, we did not include this taxon in the present analysis). The humerus here de-scribed adds some new data on forelimb morphol-ogy of this group of ceratosaurian theropods.

MCF-PVPH 53 is interpreted as belonging to an abelisauroid theropod, as supported by the presence of a proximally inflated humeral head, a reduced deltopectoral crest, greater tubercle located at level with medial tuberosity, and tuberosity on caudal surface of humeral shaft (all these features are present in Elaphrosaurus; $\mathrm{O}$. Rauhut, pers. comm.). Additionally, the humerus described here is not assigned to Abelisauridae because it retained a strongly reduced medial tuberosity that contrasts with the well developed one of Carnotaurus and Aucasaurus. The condi- 
tion of the humeral head in MCF-PVPH 53 looks more primitive than in Elaphrosaurus, Masiakasaurus, Aucasaurus and Carnotaurus, because the first one lacks a rounded humeral head (in proximal aspect), a greater tubercle that is clearly offset from the humeral head (O. Rauhut, pers. comm.), and a deep cranial ligament sulcus. In this context, MCF-PVPH 53 seems to be one of the most basal abelisauroids, even more basal than the Jurassic Elaphrosaurus. MCF-PVPH 53 opens a new panorama regarding to the radiations of basal members of Abelisauroidea, where stemabelisauroids survived until late Turonian-early Coniacian times, thus depicting a currently cov-ered radiation of basal abelisauroids from Middle Jurassic times, at least (as it is indicated by Elaphrosaurus), up to the Late Cretaceous.

Among Abelisauroidea two differerent kinds of humeri can be recognized: a plesiomorphic, gracile and elongate morphotype represented by Masiakasaurus and MCF-PVPH 53, and a shorter and stouter kind of humerus as present in abelisaurids (e.g., Aucasaurus and Carnotaurus). Curiously, they are reminiscent of the humerus of ornithomimosaurs and tyrannosaurids, respectively, in which the deltopectoral crest is proximodistally reduced and subtriangular in side view (e.g., Gauthier, 1986; Rauhut, 2003). Moreover, in Tyrannosaurus (Brochu, 2002) the humeral head is also proximally inflated, resembling the condition present in abelisauroids.

The non- abelisaurid abelisauroid condition of MCF-PVPH 53 indicates that during the deposition of the Portezuelo Formation two kinds of abelisauroid theropods co-existed. On the one hand, the large-sized and bulky abelisaurid Ekrixinatosaurus ; on the other hand, a smaller and gracile basal abelisauroid (MCF-PVPH 53). Thus, the abelisauroid faunistic component of Portezuelo Formation resembles to that of other Cretaceous Gondwanan outcrops (e.g., India, Madagascar), which shows the simultaneous presence of large-sized abelisaurids (Indosuchus, India; Majungatholus, Madagascar) and smaller non-abelisaurid abelisauroid theropods (Laevisuchus, India; Masiakasaurus, Madagascar).

\section{ACKNOWLEDGEMENTS}

The comments provided by $\mathrm{O}$. Rauhut and $\mathrm{S}$. Archangelsky are greatfully appreciated, which improved the quality of the manuscript. Agustina Lecuona took some of the photographs that composed the figures of the present contribution. Rodolfo A. Coria lended the material under his care for study.

\section{BIBLIOGRAPHY}

Baumel, J. J \& R. J. Raikow. 1993. Arthrologia. In: J. J. Baumel, A. King, J. Breazile, H. Evans \& J. Vanden Berge (eds.), Handbook of Avian Anatomy: Nomina Anatomica Avium Vol. 23, pp. 133-187.

Bonaparte, J. F. 1985. A horned Cretaceous carnosaur from Patagonia. Nat. Geo. Research 1: 149-151. 1986. History of the terrestrial Cretaceous vertebrates of Gondwana. IV Congreso Argentino de Paleontología y Bioestratigrafía. Actas II: 63-95.

1991. The Gondwanan theropod families Abelisauridae and Noasauridae. Historical Biology 5: 1-25.

Bonaparte, J. F. \& F. E. Novas. 1985. Abelisaurus comahuensis, $n$. gen., $n$. sp., Carnosauria del Cretácico Tardío de Patagonia. Ameghiniana 21: 259- 265.

Bonaparte, J. F., F. E. Novas \& R. A. Coria. 1990. Carnotaurus sastrei Bonaparte, the horned, lightly built carnosaur from the Middle Cretaceous of Patagonia. Contributions in Science, Natural History Museum of Los Angeles County 416: 1-41.

Brochu, C. A. 2002. Osteology of Tyrannosaurus rex: insights from a nearly complete skeleton and highresolution computed tomography analysis of the skull. Mem. Soc. Vert. Paleontol. 7: 1-138.

Calvo, J. O., D. Rubilar -Rogers \& K. Moreno. 2004a. A new Abelisauridae (Dinosauria: Theropoda) from northwest Patagonia. Ameghiniana 41: 555-564

Calvo, J. O., J. D. Porfiri, C. Veralli, F. E. Novas \& F. Poblete. 2004b. Phylogenetic status of Megaraptor namunhuaiquii Novas based on a new specimen from Neuquén, Patagonia, Argentina. Ameghi-niana 41: 565-576.

Calvo, J. O., J. D. Porfiri \& A. W. A Keller. 2004. On a new maniraptoran dinosaur (Theropoda) from the Upper Cretaceous of Neuquen, Patagonia, Argentina. Arq. Mus. Nac., Rio de Janeiro, 62: 549-566.

Carrano, M. T., S. D. Sampson \& C. A. Forster. 2002. The osteology of Masiakasaurus knopfleri, a small abelisauroid (Dinosauria: Theropoda) from the Late Cretaceous of Madagascar. J. Vert. Paleontol. 22: 510-534.

Coria, R. A. \& P. Currie. 2002. Un gran terópodo celurosaurio en el Cretácico Superior de Neuquén. Ameghiniana 39: 9R.

Coria, R. A., L. M. Chiappe \& L. Dingus. 2002. A new close relative of Carnotaurus sastrei Bonaparte 1985 (Theropoda: Abelisauridae) from the Late Cretaceous from Patagonia. J. Vert. Paleontol. 22: 460-465

Currie, P. J. \& K. Carpenter. 2000. A new specimen of Acrocanthosaurus atokensis (Theropoda, Dinosauria) from the Lower Cretaceous Antlers Formation (Lower Cretaceous, Aptian) of Oklahoma, USA. Geodiversitas 22: 207-246.

Galton, P. M. 1982. Elaphrosaurus, an ornithomimid dinosaur from the Upper Jurassic of North America and Africa. Paläontologische Zeitschrift 56: 265-275.

Gauthier, J. 1986. Saurischian monophyly and the origin of birds. In: K. Padian (ed.), The Origin of Birds and the Evolution of Flight, Mem. California Ac. Sc. 8: 1-55. 
Holtz, T. R. 1994. The phylogenetic position of the Tyrannosauridae: implications for theropod systematics. J. Paleontol. 68: 1100-1117.

2000. A new phylogen
saurs. Gaia 15: 5-61.

Janensch, W. 1920. Ueber Elaphrosaurus bambergi und die Megalosaurier aus den TendaguruSchichten Deutsch Ostafrikas. Sitz. Ber. Naturforsch. Fr. Ber-lin: 225-235.

Leanza, H. A., S. Apesteguia, F. E. Novas \& M. S. de la Fuente. 2004. Cretaceous terrestrial beds from the Neuquén Basin (Argentina) and their tetrapod assemblages. Cretaceous Research 25: 27 pp.

Madsen Jr., J. H. 1976. Allosaurus fragilis: a revised osteology. Utah Geol. Min. Surv. Bull. 109: 1-163.

Madsen Jr., J. H. \& S. P. Welles. 2000. Ceratosaurus (Dinosauria, Theropoda) a Revised Osteology. Miscellaneous Pub. 00-2 Utah Geol. Surv.: 1-80.

Novas, F. E. 1997. Anatomy of Patagonykus puertai (Theropoda, Avialae, Alvarezsauridae), from the Late Cretaceous of Patagonia. Jour. Vert. Paleont. 17: 137-166.

- $\quad$ 1998. Megaraptor namunhuaiquii gen. et. sp. nov., a large-clawed, Late Cretaceous Theropod from Argentina. Jour. Vert. Paleont.18: 4-9.

Novas, F. E. \& P. Puerta. 1997. New evidence concerning avian origins from the Late Cretaceous of Patagonia. Nature 387: 390-392.
Novas, F. E. \& D. Pol. 2005. New evidence of deinonychosaurian dinosaurs from Late Cretaceous of Patagonia. Nature 433: 858-861.

Porfiri, J. D., J. O. Calvo \& F. E. Novas. 2005. Hallazgo de un nuevo Theropoda del Cretácico Tardío en Lago Barreales, Neuquén, Patagonia, Argentina. II Congreso Latino-Americano de Paleontología: 209-210.

Raath, M. A. 1977. The anatomy of the Triassic theropods Syntarsus rhodensiensis (Saurischia: Podokesauridae) and a consideration of its biology. Ph. D. dissertation, Rhodes University, Salisbury, $233 \mathrm{pp}$.

Rauhut, O. W. M. 2003. The interrelationships and evolution of basal theropod dinosaurs. Special papers in Paleontology 69: 1-214.

Sereno, P. C. 1993. The pectoral girdle and forelimb of the basal theropod Herrerasaurus ischigualastensis. J. Vert. Paleontol. 13: 425-450.

Sereno, P. C., D. B. Dutheil, M. larochene, H. C. E. Larsson, G. H. Lyon, P. M. Magwene, C. A. Sidor, D. J. Varricchio \& J. A. Wilson. 1996. Predatory dinosaurs from the Sahara and Late Cretaceous faunal differentiation. Science 272: 986-991.

Sereno, P. C., J. A. Wilson \& J. L. Conrad. 2004. New dinosaurs link landmasses in the Mid-Cretaceous. Proc. R. Soc. Lond. B: 1-6.

Welles, S. P. 1984. Dilophosaurus wetherilli (Dinosauria, Theropoda): osteology and comparisons. Palaeontographica Abteilung A 185: 85-180. 